\title{
Ultrafast Electron Mircoscopy: The Problem of Spatial Coherence
}

\author{
W. Andreas Schroeder
}

Department of Physics, University of Illinois at Chicago (m/c 273), 845 W. Taylor Street, Chicago, IL 60607-7059

Several efforts [1-3] are currently underway to develop an ultrafast electron microscope (UEM): that is, to combine the atomic-scale resolution of electron microscopy with the sub-picosecond $\left(<10^{-12} \mathrm{~s}\right)$ temporal resolution now standard in ultrafast laser spectroscopy. In such an instrument, an ultrashort (ideally femtosecond) pump laser pulse initiates a dynamic transient event in a sample that is observed and time-resolved by a synchronized sub-ps electron pulse (Figure 1). The resulting unprecedented spatio-temporal resolution could allow for the direct visualization of the dynamic properties of individual nanoparticles and, in the future, individual atomic motions, thus providing an important new tool for sensing materials and molecular dynamics on the nanoscale [3]. To date, however, UEMs have operated almost exclusively as a dynamic transmission electron microscope (DTEM) in the diffraction mode; i.e.,monitoring ensemble atomic motions in the Fourier plane as in ultrafast electron diffraction [4]. The inability to produce high spatial resolution images in a UEM can be traced to the lack of adequate spatial coherence in the electron pulse.

Ideally, in a UEM, the quantum state function $\Psi$ for $N$ individual electrons propagating in different directions $\mathbf{k}_{j}$ with different energies $E_{j}=\hbar \omega_{j}$ and phases $\phi_{j}$, should tend to that of a pulse propagating in one direction $k$, with one energy $E=\hbar \omega$, and with a well defined (i.e., spatially phase-coherent) amplitude $\Psi_{0}(\mathbf{r}, t)$; that is

$$
\Psi=\sum_{j=1}^{N} \exp \left[i\left(\mathbf{k}_{j} \cdot \mathbf{r}-\omega_{j} t+\phi_{j}\right)\right] \approx \Psi_{0}(\mathbf{r}, t) \exp [i(k z-\omega t)] .
$$

The problem is that the electrons photoemitted from the laser-driven photocathode, in general, will not be generated with the same energy, wave vector k, and phase. Moreover, even if a spatiallycoherent electron pulse is emitted from the photocathode, space-charge effects (i.e., repulsive electron-electron scattering) will destroy the spatial coherence [2] and temporally broaden the pulse $[5,6]$. While the latter effect could be compensated for by the use of a synchronized RF cavity (Figure 1), the destruction of the spatial coherence will be detrimental to the spatial resolution of a UEM. Fortunately, a detailed numerical investigation into the space-time dynamics of electron pulses has revealed that for 'disk-like' pulses (i.e., transverse radial dimension much larger than the length of the pulse in the propagation direction) space-charge effects predominantly affect the temporal duration of the electron pulse upon propagation down the column. Thus, if the electron pulse can be photoemitted with a degree of spatial coherence, there is a good chance that electronelectron scattering will not substantially degrade the spatial coherence over the first few nanoseconds of propagation time.

Figure 2 illustrates the method that is currently being investigated as a means to generate a spatially coherent electron pulse form a nano-patterned photocathode. An array of silicon nanoposts (field emitters) is irradiated by an intense ultrashort laser pulse in such a manner to ensure that the phase of the incident laser field is the same at each nano-emitter. The resulting 'phase-control' of the photoemission could yield a set of coherent Huygen's electron wavelets to constitute an initially spatially coherent electron pulse required for the UEM (Figure 1) to achieve sub-nm resolution [7]. 
Fig. 1. Schematic of the proposed UEM operated in transmission mode. The output of the high-power, infra-red (IR), femtosecond laser is partially converted to the ultraviolet (UV) in a harmonic generator $(\mathrm{HG})$ to produce the pulsed laser radiation required for the spatially-coherent photoemission from the nano-patterned photocathode. A RF cavity, synchronized to the $10-100 \mathrm{MHz}$ laser repetition frequency, compensates for any space-charge-induced broadening of the electron pulse prior to it being focused onto the sample using a magnetic lens. Detection of the transmitted electron 'probe' pulse then allows the temporal resolution of a perturbation initiated by a time-delay controlled residual IR pulse form the laser.

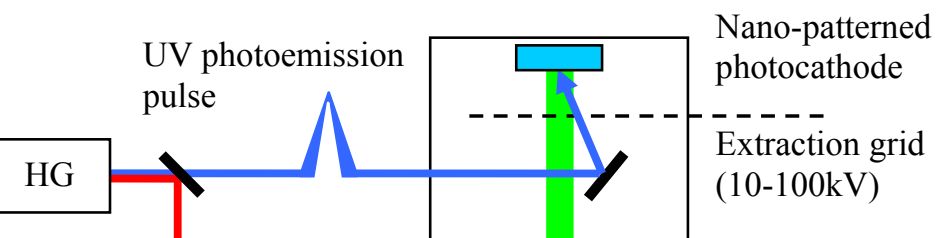

(10-100kV)

Synchronized

RF cavity

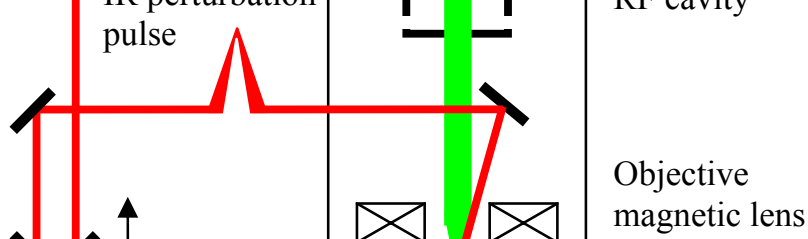

Sample

Time delay, $\tau$

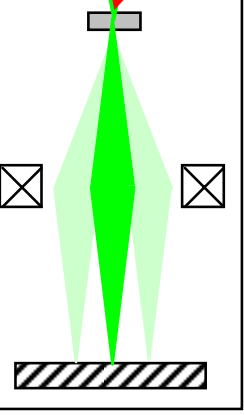

Imaging magnetic

lenses

Detectors

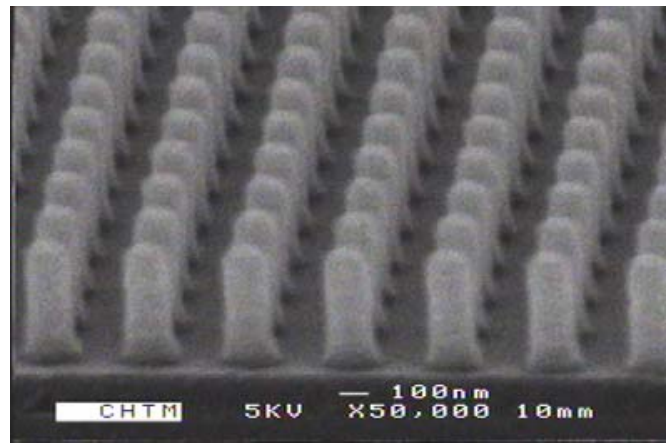

(a)

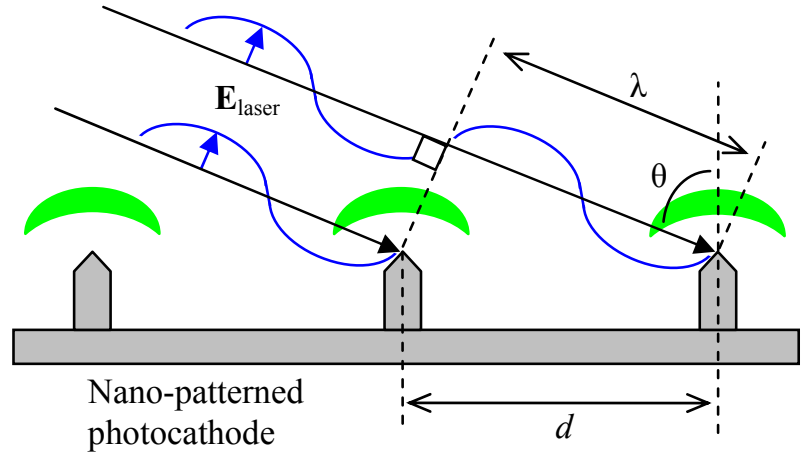

(b)

Fig. 2. (a) Cross-section SEM image of an array of $110 \mathrm{~nm}$ silicon nanoposts with a pitch of $360 \mathrm{~nm}$ manufactured using laser interference lithography (courtesy of R. Bommena, Universty of New Mexico).

(b) Schematic of the irradiance condition $(\lambda=d \sin \theta)$ at wavelength $\lambda$ proposed to achieve laser-driven phasecoherent electron photoemission (green wavelets) from a nano-patterned photocathode with spatial period $d$.

\section{References}

[1] O. Bostanjoglo et al., Ultramicroscopy 81 (2000) 141.

[2] V.A. Lobastov et al., PNAS 102 (2005) 7069.

[3] W.E. King, et al., J. Appl. Phys. 97 (2005) 111101.

[4] See, for example, J. Cao et al., Appl. Phys. Lett. 83 (2003) 1044.

[5] H. Niu and W. Sibbett, Rev. Sci. Instrum. 52 (1981) 1830.

[6] B.J. Siwick et al., J. Appl. Phys. 92 (2002) 1643.

[7] This research is supported by the National Science Foundation under award NER-0508143. 